\title{
Frecuencia y Caracterización de Foramen Mental Accesorio en una Población Chilena Mediante Tomografía Computarizada de Haz Cónico
}

\author{
Frequency and Characterization of Accessory Mental Foramen in \\ a Chilean Population Using Cone Beam Computed Tomography
}

\author{
Javiera Barría-Pérez ${ }^{1}$; Hernán Castillo-Cubillos ${ }^{1}$; Cristian Rosas-Méndez²; \\ Fernando Santana²; Guillermo Araya $^{2}$ \& Scarlette Hernández-Vigueras ${ }^{2}$
}

BARRÍA-PÉREZ, J.; CASTILlO-CUBILlOS, H.; ROSAS-MÉNDEZ, C.; SANTANA, F.; ARAYA, G. \& HERNÁNDEZVIGUERAS, S. Frecuencia y caracterización de foramen mental accesorio en una población chilena mediante tomografía computarizada de haz cónico. Int. J. Morphol., 39(5):1296-1301, 2021.

RESUMEN: En la práctica clínica odontológica, reconocer estructuras anatómicas es determinante en la planificación de distintos tratamientos que involucren algún riesgo. Uno de los exámenes complementarios recomendados para la planificación de diferentes tratamientos en odontología es la Tomografía Computarizada de Haz Cónico (CBCT). Con este examen, se pueden pesquisar variantes anatómicas como el Foramen Mental Accesorio (FMA), el cual es importante identificar para prevenir complicaciones. El objetivo de este trabajo fue determinar la frecuencia y caracterización de FMA en una población chilena adulta, de la ciudad de Valdivia, Chile. Este estudio es de tipo observacional descriptivo. Se revisaron 247 exploraciones de diferentes centros radiológicos de la ciudad de Valdivia, cumpliendo 143 con los criterios de selección. Este estudio fue aprobado por el Comité ético científico del Servicio de Salud de Valdivia. Se encontró presencia de FMA en el 17,48 \% que corresponde a 25 pacientes de un total de 143, correspondiendo un $80 \%$ al sexo femenino. El rango etario en que se encontró con mayor frecuencia correspondió a 18-39 años. La distancia promedio entre FMA y FM (Foramen Mental) fue de $5,76 \mathrm{~mm}$, correspondiendo a $2 \mathrm{~mm}$ la distancia mínima y 11,5 $\mathrm{mm}$ la distancia máxima. La distancia promedio entre FMA y ápice dentario más cercano fue de $5,36 \mathrm{~mm}$. La distancia mínima y máxima fueron de 0,8 mm y 10,2 mm respectivamente. El ápice radicular mayormente asociado al FMA correspondió al segundo premolar con una frecuencia de $60 \%$ ( $\mathrm{n}=15$ ). Este estudio confirma la importancia de la correcta evaluación de la región mental ante procedimientos en la zona, la cual es vital para prevenir lesiones en relación a esta variante anatómica.

PALABRAS CLAVE: Daño Nervio Mandibular; Daño Mandibular; Tomografía computacional de haz cónico; Foramen Mental Accesorio; Foramen Mental.

\section{INTRODUCCIÓN}

El Foramen Mental Accesorio (FMA) es una variante anatómica, la cual presenta una descripción variada. Corresponde a un foramen de menor tamaño con respecto al Foramen Mental (FM), que emerge como una ramificación nerviosa en el bucle antes de su salida por el FM (Kaufman et al., 2000). Por otra parte, también se describe el FMA integrado al canal mandibular (Katakami et al., 2008; Naitoh et al., 2009).

Su importancia radica principalmente para la planificación de cirugía de implantes, cirugía periodontal, tratamientos endodónticos y cirugía periapical, corres- pondiendo a un descubrimiento inesperado. En ese sentido, se genera un problema, ya que esta estructura anatómica no se profundiza en la enseñanza de pregrado y además, es de difícil hallazgo en radiografías panorámicas (Iwanaga et al., 2016).

El diagnóstico imagenológico mediante $\mathrm{CBCT}$ permite obtener mayor precisión en la detección y caracterización de FMA, debido a sus características imagenológicas en tres dimensiones. El examen complementario con mayor comparación dentro de los estudios asociados a FMA es la radiografía panorámica, la cual se

\footnotetext{
${ }^{1}$ Escuela de Odontología, Facultad de Medicina, Universidad Austral de Chile, Valdivia, Chile. Los autores comparten la primera autoría.

${ }^{2}$ Instituto de Odontoestomatología, Facultad de Medicina, Universidad Austral de Chile, Valdivia, Chile.
} 
BARRÍA-PÉREZ, J.; CASTILLO-CUBILLOS, H.; ROSAS-MÉNDEZ, C.; SANTANA, F; ARAYA, G. \& HERNÁNDEZ-VIGUERAS, S. Frecuencia y caracterización de foramen mental accesorio en una población chilena mediante tomografía computarizada de haz cónico. Int. J. Morphol., 39(5):1296-1301, 2021.

concluye que no es adecuada para identificar apropiadamente el FMA. Es por esto, que se recomienda el uso de CBCT para estudios prequirúrgicos en la zona (MuineloLorenzo et al., 2015). Se comparó la detección de FMA mediante radiografía panorámica y CBCT, concluyendo que la radiografía panorámica detectó solo 48,6 \% de FMA de todos los detectados por el CBCT (Naitoh et al., 2011). Conocer la existencia de esta estructura anatómica es fundamental, ya que su detección previa puede reducir el riesgo de complicaciones, como hemorragia, dolor postoperatorio y parálisis (Goyushov et al., 2017).

La prevalencia observada de FMA varía entre 1,4 $\%$ al $20 \%$ (Li et al., 2018), existiendo una falta de consenso en los estudios publicados (Aytugar et al., 2019). Al respecto, se reporta una alta heterogeneidad en los datos debido a diferencias étnicas, distintas definiciones de FMA y por el número de pacientes evaluados en los estudios (Han et al., 2016). En este sentido, se menciona que las etnias no caucásicas tienen mayor prevalencia de FMA que las caucásicas (Balcioglu \& Kocaelli, 2009).

Es por esto, que existe interés en determinar la frecuencia y caracterización de FMA en población adulta chilena, dada la necesidad de conocer la existencia de esta estructura anatómica para planificaciones óptimas de tratamientos clínicos y quirúrgicos en ausencia de complicaciones.

El objetivo de este estudio fue determinar la frecuencia y caracterización de FMA en una población chilena adulta, de la ciudad de Valdivia, Chile, 2021.

\section{MATERIAL Y MÉTODO}

Se realizó un estudio descriptivo utilizando exámenes de CBCT, para determinar frecuencia y caracterización del FMA, en una población chilena adulta, de la ciudad de Valdivia, Chile. El estudio cuenta con la aprobación del Comité Ético Científico del Servicio de Salud de Valdivia, Chile (Ord 415).

Se incluyeron 143 CBCT que cumplieron con los siguientes criterios de inclusión: exámenes de pacientes con edad igual o mayor a 18 años, CBCT mandibular y/o bimaxilar que estén registrados en alguno de los dos centros radiológicos maxilofacial privados de la ciudad de Valdivia, que participaron del estudio, y ausencia de artefactos que alteren visibilidad en la región de estudio. Los criterios de exclusión fueron: CBCT repetidos del mismo paciente donde se consideró el más reciente (uti- lizando el RUT como medio identificador), pacientes desdentados en región de estudio, pacientes con reabsorción ósea que alteren visibilidad en la región de estudio, presencia de patologías óseas que alteren visibilidad en la región de estudio y presencia de cirugías mentonianas.

En cuanto a la presencia de patología ósea se consideró todo aquel paciente que presente una lesión (radiolúcida o radiopaca) en relación al FM o ápice dentario, y para los CBCT no diagnóstico se consideraron aquellos que presenten artefactos metálicos que distorsionen la imagen.

Se examinaron los CBCT realizados en el periodo comprendido entre enero del 2018 a marzo del 2021, en pacientes adultos, atendidos en dos centros de radiología maxilofacial privados de la ciudad de Valdivia, Chile.

Para asegurar la validez de los resultados todas las muestras se vieron en conjunto entre cuatro de los investigadores, y se llegó a acuerdo en todos los casos.

Los exámenes de CBCT fueron observados mediante el visualizador del software Carestream en los siguientes equipos: Cone Beam CS8100 3D (Carestream, Atlanta, USA) con las siguientes especificaciones: imágenes escaneadas mediante parámetros de exposición de 90 kV y 4 mA, vóxel de 75 um y FOV (campo de visión) con diámetro estándar de $8 \times 9 \mathrm{~cm}$ (máximo) y un tiempo de exposición aproximado de 14 segundos.

Cone Beam Orthophos XG3D (Sirona, Bensheim, Alemania) con las siguientes especificaciones: imágenes escaneadas mediante parámetros de exposición de $60 \mathrm{kV}$ 90 kV y 4 mA, vóxel de 100 um y FOV (campo de visión) con diámetro estándar de $8 \times 9 \mathrm{~cm}$ (máximo) y un tiempo de exposición aproximado de 14 segundos.

Se analizaron reconstrucciones de imágenes tridimensionales ocupando cortes transversales y axiales, a partir de datos volumétricos de CBCT, utilizando el Software Cs imaging 7 (Carestream, Atlanta, USA) para la visualización y análisis de los exámenes CBCT. Los exámenes CBCT tomados con Cone Beam Orthophos XG3D, fueron importados en formato DCM para análisis en el visualizador.

Se analizó la reconstrucción 3D de cada examen, luego en caso de sospecha de FMA, se realizó curva panorámica y se revisaron cortes transversales con $1 \mathrm{~mm}$ de grosor y $1 \mathrm{~mm}$ de intervalo de corte, en un rango de 10 $\mathrm{mm}$ dejando al centro el foramen mental. 
Para medir la distancia desde FMA hacia FM se utilizó el corte oblicuo y para la medición desde FMA hacia ápice vestibular más cercano, se utilizó el corte curvo.

Se dejó el tamaño de vóxel lo más pequeño posible (150 micras) al momento de hacer mediciones.

Se categorizó a la población según sexo: Femenino/ Masculino y según grupo etario: 18 - 39 años, 40 - 59 años y >60 años. Así mismo, se evaluó la presencia/ ausencia de FMA, localización según lado mandibular de FMA: derecho, izquierdo y bilateral. Además las distancias obtenidas, fueron determinadas según ubicación desde el FM, en donde se trazó una línea recta entre las superficies más próximas de FM y FMA, y se obtuvieron medidas en milímetros; y también según distancia desde ápice radicular, en donde se trazó una línea recta desde la cara más próxima del ápice radicular vestibular más cercano hacia el FMA.

Los datos se analizaron mediante el programa Rstudioversion 1.2.1335, (RStudio PBC, Boston, USA), mediante estadística de frecuencias para las variables nominales y estadística descriptiva para las variables continuas, obteniendo promedio y desviación estándar (DE).

\section{RESULTADOS}

De un total de 247 exploraciones CBCT, se seleccionaron 143 CBCT que cumplieron con los criterios de inclusión y exclusión. De las cuales un 28,67 \% $(n=41)$ corresponden a CBCT masculinos y $71,32 \%(n=102)$ femeninos, con un rango de 18 a 79 años de edad.

La frecuencia encontrada de FMA fue de 17,48 \% $(n=25)$, de los cuales corresponden $20 \%(n=5)$ al sexo masculino y $80 \%(n=20)$ al sexo femenino. El desglose de los resultados en relación a rango etario y sexo se encuentran en la Tabla I.

En cuanto a localización según lado mandibular, se encontró un $40 \%(n=10)$ en lado derecho, $44 \%(n=11)$ lado izquierdo y $16 \%(n=4)$ bilateralmente. La cantidad máxima de FMA encontrada en un solo individuo fue de 4, presentando 1 en el lado derecho y 3 en el lado izquierdo (Fig. 1).

El cálculo de distancia entre FM y FMA, determinó como distancia mínima 2,0 mm, como distancia máxima $11,5 \mathrm{~mm}$ y un promedio de $5,76 \mathrm{~mm}$ con una DE de 1,6 mm. Las mediciones obtenidas entre FMA y los ápices dentarios más cercanos se encuentran en la Tabla II.
Tabla I. Distribución del FMA visualizado en CBCT según sexo y edad.

\begin{tabular}{lll}
\hline Variables & Subgrupo & Casos $(\%)$ \\
\hline Sexo & Femenino & $20(80)$ \\
& Masculino & $5(20)$ \\
Rango etario & $18-39$ & $18(72)$ \\
& $40-59$ & $7(28)$ \\
& $>60$ & $0(0)$ \\
\hline
\end{tabular}

Tabla II. Distancias mínima, máxima y promedio entre FMA y ápice.

\begin{tabular}{lcccc}
\hline & $\begin{array}{c}\text { Distancia } \\
\text { min. } \\
\text { FMA }(\mathrm{mm})\end{array}$ & $\begin{array}{c}\text { Distancia } \\
\text { máx. } \\
\text { FMA }(\mathrm{mm})\end{array}$ & $\begin{array}{c}\text { Promedio } \\
(\mathrm{mm})\end{array}$ & $\begin{array}{c}\text { DE } \\
(\mathrm{mm})\end{array}$ \\
\hline $1^{\circ}$ Premolar & 4,7 & 10,2 & 6,9 & 2,1 \\
$2^{\circ}$ Premolar & 0,8 & 8,4 & 4,7 & 1,6 \\
$1^{\circ}$ Molar & 3,5 & 6,9 & 5,4 & 0,7 \\
\hline
\end{tabular}



Fig. 1. Presencia de cuatro FMA en una paciente del estudio, tres en el lado izquierdo mandibular, y uno en el derecho.

\section{DISCUSIÓN}

El análisis de los resultados de este estudio descriptivo determinó que la frecuencia de FMA en la población chilena adulta de la ciudad de Valdivia fue del 17,48 $\%$, siendo mayor a la esperada. La distancia mínima, máxima y media entre FMA y ápice dentario fue de $0,8 \mathrm{~mm}$, $10,2 \mathrm{~mm}$ y $5,36 \mathrm{~mm}$ respectivamente. Siendo el segundo premolar mandibular el diente más frecuentemente asociado con un $60 \%$.

En el caso del FM, en el estudio de AndradeAlvarado et al. (2020) en una población similar a la de este estudio, se determinó que la distancia promedio encontrada entre FM y el ápice más cercano fue de 3,22 mm, la cual es levemente menor a la distancia encontrada en nuestro estudio, entre FMA y ápice más cercano. En am- 
bos estudios, el ápice dentario más cercano tanto al FM y a FMA, es del segundo premolar inferior. En el mismo estudio, se encontró que la menor distancia entre FM y ápice más cercano fue de $0,81 \mathrm{~mm}$, resultado similar a la menor distancia entre FMA y el ápice dentario más cercano obtenido en el presente estudio (Andrade-Alvarado et al.).

Los resultados en relación a la frecuencia de FMA, son similares a los reportados en otros, donde varía entre el $1,4 \%$ al $20 \%$ (Li et al.). En la ciudad de Valparaíso, Chile, un estudio presentó una frecuencia de 3,6\% con respecto al FMA, encontrándose en todos los casos de forma unilateral (Pezoa et al., 2013). Sin embargo, este último estudio fue realizado con tomografía axial computarizada (TC de uso médico), lo cual puede motivar la menor frecuencia, ya que el CBCT al tener un vóxel más pequeño, es más factible distinguir estructuras anatómicas de menor tamaño versus TC médico.

En cuanto a la frecuencia de FMA según sexo, se mencionan diferencias estadísticamente significativas, lo que difiere de los resultados obtenidos en otros estudios (Han et $a l . ;$ Li et al.). En nuestro estudio, no es posible establecer esta relación, ya que de los exámenes que cumplieron los criterios de selección, corresponden en su mayoría a pacientes mujeres, con un $71 \%$.

La ubicación más frecuente del FMA es entre primer y segundo premolar, resultados similares a los encontrados en nuestro estudio (Kalender et al., 2012; Han et al.; Li et $a l$.). En cambio, en otro estudio hubo mayor frecuencia entre segundo premolar y primer molar (Oliveira-Santos et al., 2011).

Uno de los casos de mayor interés en este estudio, fue de una paciente con un total de 4 FMA (Fig. 1), con 3 FMA en lado izquierdo y 1 FMA en lado derecho, sin contar los FM principales. La aparición de FMA se inicia en el desarrollo embrionario, antes de la formación del foramen en la duodécima semana de gestación (Lam et al., 2019). Por un lado, el FM principal está formado por un aumento de aposición ósea de haces nerviosos, antes de la ramificación del nervio mandibular cuando sale del FM. Por otra parte, el FMA está formado por una aposición ósea después de la ramificación, por consiguiente, las características del FMA dependen tanto del grosor del haz de nervio, como también de la cantidad de ramificaciones (Iwanaga et al.).

Las diferencias entre los grupos étnicos no fueron evaluadas en nuestro estudio. No obstante, si existe evidencia variable sobre las diferencias étnicas, se informan las siguientes frecuencias de FMA: 2,6 \% Francia; 1,4 \% Americanos Blancos; 5,7 \% Afroamericanos; 3,3\% Griegos; 1,5
$\%$ en Rusia; 3,0 \% en Hungría; 9,7 \% en Melanesia y 3,6 \% en Egipto (Sawyer et al., 1998). También se señalan que etnias de África subsahariana y Asia central tienen una frecuencia sobre $20 \%$ (Hanihara \& Ishida, 2001). En una población japonesa los estudios realizados mostraron que la frecuencia del FMA oscila entre el 6,7 \% y $12,5 \%$. Esto revela que los no caucásicos pueden tener una mayor incidencia de FMA versus caucásicos (Toh et al., 1992).

En relación a la prevalencia según su ubicación mandibular, en nuestro estudio se encontró un $40 \%(n=10)$ en lado derecho y $44 \%(\mathrm{n}=11)$ en lado izquierdo, en comparación con el estudio de Zmyslowska-Polakowska et al. (2017) donde correspondieron 53,6 \% ( $\mathrm{n}=15)$ en lado derecho y $46,4 \%(n=13)$ en el lado izquierdo, es decir, se obtienen resultados similares en el sentido de que no se encuentran grandes diferencias a los valores obtenidos por lado.

El diagnóstico de FMA mediante una adecuada evaluación imagenológica, para la planificación de procedimientos que involucren la zona mentoniana, es relevante para evitar daño en estructuras nerviosas, disminuyendo la tasa de hemorragia y parestesia en la región. Se debe tener precaución ya que, en radiografías convencionales, como la periapical, el FMA puede presentarse como una radiolucidez periapical, lo que podría llevar a un diagnóstico erróneo (Iwanaga et al.; Borghesi et al., 2018). Además, se ha encontrado que después de una sobreobturación endodóntica y cirugía, ha existido relación con parestesia de labio y mandíbula (Katakami et al.).

Dentro de los reportes de casos encontrados, se presenta uno donde se realizó neurectomía periférica del nervio mental derecho, donde se descubrió FMA durante la disección subperióstica. La omisión de la rama accesoria en la salida del FMA, hubiera significado recurrencia del dolor y fracaso del tratamiento. Para evitar complicaciones neurovasculares, el clínico debe tener cautela durante procedimientos, en particular, de colocación de implantes, anestesia regional, corrección quirúrgica mandibular y cirugía periapical (Thakur et al., 2011).

Anteriormente, se han utilizado radiografías panorámicas e intraorales para evaluar FM y sus variaciones. Hoy en día, se sabe que no son completamente confiables, ya que el FMA no es comúnmente visible en imágenes bidimensionales, debido a la proyección geométrica e inherente distorsión. Es por esto, que el CBCT es el método más indicado y utilizado, por tener mayor resolución ósea espacial y menor dosis de radiación que la tomografía computarizada médica (Lam et al.). Además, el uso de la tomografía computarizada médica es discutible, puesto que comúnmente tienen un tamaño de vóxel más grande, y al 
ser mayor, no se visualizan estructuras finas como conductos accesorios de la manera en que se visualiza en el CBCT.

Los datos de este estudio, refuerzan la importancia de la planificación adecuada para los tratamientos odontológicos y/o quirúrgicos en la zona, buscando minimizar el riesgo de daño de estructuras en la zona, con sus posibles consecuencias posteriores.

El estudio del FMA debe ser considerado por parte del clínico para brindar cada vez, tratamientos más predecibles, con un desempeño óptimo ante los desafíos que involucra la región mental.

\section{AGRADECIMIENTOS}

Esta investigación está basada en parte de los requerimientos de Javiera Catalina Barría Pérez y Hernán Domingo Castillo Cubillos para su titulación de Cirujano-Dentista en la Escuela de Odontología de la Universidad Austral de Chile.

Se agradece al Comité Ético Científico Servicio de Salud Valdivia por el oficio de la aprobación del estudio.

BARRÍA-PÉREZ, J.; CASTILLO-CUBILLOS, H.; ROSASMÉNDEZ, C.; SANTANA, F.; ARAYA, G. \& HERNÁNDEZVIGUERAS, S. Frequency and characterization of accessory mental foramen in a Chilean population using Cone Beam Computed Tomography. Int. J. Morphol., 39(5):1296-1301, 2021.

SUMMARY: The recognition of key anatomical structures is decisive to avoid complications in the dental clinical practice. Cone Beam Computed Tomography (CBCT) is a complementary exam recommended for the planning of different procedures in dentistry. With this exam, anatomical variants can be identified, such as the Accessory Mental Foramen (AMF). The objective of this work was to determine the frequency and characteristics of AMF in an adult Chilean population from the city of Valdivia, Chile. This is an observational study that included 247 CBCT exams from different radiological centers in Valdivia. According to the selection criteria, 143 CBCT exams were included. This study was approved by the Scientific Ethics Committee of the Valdivia Health Services. AMF was identified in 25 patients representing $17.48 \%$. From this result, $80 \%$ were found in females. AMF was most frequently identified in patients from 18 to 39 years old. The average distance between AMF and Mental Foramen (MF) was $5.76 \mathrm{~mm}$, which corresponds to $2 \mathrm{~mm}$ the minimum distance and $11.5 \mathrm{~mm}$ the maximum distance. The average distance between AMF and the nearest dental apex was $5.36 \mathrm{~mm}$, and the minimum and maximum distance were $0.8 \mathrm{~mm}$ and $10.2 \mathrm{~mm}$, respectively. The root apex of the second premolar was most frequently associated with the AMF, representing $60 \%(n=15)$. This study confirms the importance of the correct evaluation of the mental region before initiating procedures in the area, which is vital to prevent injuries associated with this anatomical variant.

KEY WORDS: Mandibular nerve injuries; Mandibular injuries; Cone-Beam Computed Tomography; Accessory mental foramen; Mental foramen.

\section{REFERENCIAS BIBLIOGRÁFICAS}

Andrade-Alvarado, S.; Jara-Calderón, R.; Sanhueza-Tobar, C.; AracenaRojas, D. \& Hernández-Vigueras, S. Anatomical localization of the mental foramen using cone-beam computed tomography in a population in Chile: observational study. Int. J. Morphol., 38(1):2037, 2020 .

Aytugar, E.; Özeren, C.; Lacin, N.; Veli, I. \& Çene, E. Cone-beam computed tomographic evaluation of accessory mental foramen in a Turkish population. Anat. Sci. Int., 94(3):257-65, 2019.

Balcioglu, H. A. \& Kocaelli, H. Accessory mental foramen. N. Am. J. Med. Sci., 1(6):314-315, 2009.

Borghesi, A.; Pezzotti, S.; Nocivelli, G. \& Maroldi, R. Five mental foramina in the same mandible: CBCT findings of an unusual anatomical variant. Surg. Radiol. Anat., 40(6):635-40, 2018.

Goyushov, S.; Tözüm, M. D. \& Tözüm, T. F. Accessory mental/buccal foramina: case report and review of literature. Implant Dent., 26(5):796-80, 2017.

Han, S. S.; Hwang, J. J. \& Jeong, H. G. Accessory mental foramina associated with neurovascular bundle in Korean population. Surg Radiol Anat., 38(10):1169-74, 2016.

Hanihara, T. \& Ishida, H. Frequency variations of discrete cranial traits in major human populations. IV. Vessel and nerve related variations. J. Anat., 199(Pt. 3):273-287, 2001.

Iwanaga, J.; Watanabe, K.; Saga, T.; Tabira, Y.; Kitashima, S.; Kusukawa, J. \& Yamaki, K. Accessory mental foramina and nerves: Application to periodontal, periapical, and implant surgery. Clin. Anat., 29(4):493$501,2016$.

Kalender, A.; Orhan, K. \& Aksoy, U. Evaluation of the mental foramen and accessory mental foramen in Turkish patients using cone-beam computed tomography images reconstructed from a volumetric rendering program. Clin. Anat., 25(5):584-92, 2012.

Katakami, K.; Mishima, A.; Shiozaki, K.; Shimoda, S.; Hamada, Y. \& Kobayashi, K. Characteristics of accessory mental foramina observed on limited cone-beam computed tomography images. J. Endod., 34(12):1441-5, 2008

Kaufman, E.; Serman, N. J. \& Wang, P. D. Bilateral mandibular accessory foramina and canals: a case report and review of the literature. Dentomaxillofac. Radiol., 29(3):170-5, 2000.

Lam, M.; Koong, C.; Kruger, E. \& Tennant, M. Prevalence of accessory mental foramina: a study of 4,000 CBCT scans. Clin. Anat., 32(8):1048-52, 2019

Li, Y.; Yang, X.; Zhang, B.; Wei, B. \& Gong, Y. Detection and characterization of the accessory mental foramen using cone-beam computed tomography. Acta Odontol. Scand., 76(2):77-85, 2018.

Muinelo-Lorenzo, J.; Suárez-Quintanilla, J. A.; Fernández-Alonso, A.; Varela-Mallou, J. \& Suárez-Cunqueiro, M. M. Anatomical characteristics and visibility of mental foramen and accessory mental foramen: panoramic radiography vs. cone beam CT. Med. Oral Patol. Oral Cir. Bucal, 20(6):e707-14, 2015.

Naitoh, M.; Hiraiwa, Y.; Aimiya, H.; Gotoh, K. \& Ariji, E. Accessory mental foramen assessment using cone-beam computed tomography. Oral Surg. Oral Med. Oral Pathol. Oral Radiol. Endod., 107(2):289-94, 2009. 
BARRÍA-PÉREZ, J.; CASTILLO-CUBILLOS, H.; ROSAS-MÉNDEZ, C.; SANTANA, F.; ARAYA, G. \& HERNÁNDEZ-VIGUERAS, S. Frecuencia y caracterización de foramen mental accesorio en una población chilena mediante tomografía computarizada de haz cónico. Int. J. Morphol., 39(5):1296-1301, 2021.

Naitoh, M.; Yoshida, K.; Nakahara, K.; Gotoh, K. \& Ariji, E. Demonstration of the accessory mental foramen using rotational panoramic radiography compared with cone-beam computed tomography. Clin. Oral Implants Res., 22(12):1415-9, 2011.

Oliveira-Santos, C.; Souza, P. H.; De AzambujaBerti-Couto, S.; Stinkens, L.: Moyaert, K.; Van Assche, N. \& Jacobs, R. Characterisation of additional mental foramina through cone beam computed tomography. J. Oral Rehabil., 38(8):595-600, 2011.

Pezoa, O. N.; Naranjo, P. R. \& Weiss, V. F. Frecuencia de variantes anatómicas en el recorrido del canal mandibular. Estudio mediante tomografía computarizada médica. Anu. Soc. Radiol. Oral Máxilo Facial Chile, 16:33-9, 2013.

Sawyer, D. R.; Kiely, M. L. \& Pyle, M. A. The frequency of accessory mental foramina in four ethnic groups. Arch. Oral Biol., 43(5):417-20, 1998.

Thakur, G.; Thomas, S.; Thayil, S. C. \& Nair, P. P. Accessory mental foramen: a rare anatomical finding. BMJ Case Rep., 2011:bcr0920103326, 2011.

Toh, H.; Kodama, J.; Yanagisako, M. \& Ohmori, T. Anatomical study of the accessory mental foramen and the distribution of its nerve. Okajimas Folia Anat. Jpn., 69(2-3):85-8, 1992.

Zmyslowska-Polakowska, E.; Radwanski, M.; Leski, M.; Ledzion, S.; Lukomska-Szymanska, M. \& Polguj, M. The assessment of accessory mental foramen in a selected polish population: a CBCT study. BMC Med. Imaging, 17(1):17, 2017.
Dirección para correspondencia:

Dra. Scarlette Hernández-Vigueras

Instituto de Odontoestomatología

Rudloff 1640

Valdivia

CHILE

E-mail: shernandez@uach.cl

Recibido : 08-06-2021

Aceptado: 15-07-2021 